\title{
Metric for a Temporal Manifold Derived from Special Relativity and Newtonian Classical Gravitational Potential
}

\author{
Rickey W. Austin
}

(PhD Candidate, NC A\&T, Greensboro, NC, USA) Senior Research

Scientist, River Song Innovations Inc, North Carolina A\&T State University

1601 E Market St. Greensboro, NC 27401

doi: 10.19044/esj.2017.v13n18p47 URL:http://dx.doi.org/10.19044/esj.2017.v13n18p47

\begin{abstract}
In a previous paper (Austin, 2017) a method for calculating time dilation from Newtonian gravitational potential provided a first order equivalence to Schwarzschild's solution to Einstein's field equations. This equivalence is for the transformation of the time component between locations when only the radial component is changed. The derivation from the previous paper will be merged with Special Relativity's kinetic energy derivation to form a metric for a Riemannian geometry. A geodesic is derived from the metric and compared to Schwarzschild's solution.
\end{abstract}

Keywords: Gravitational Time Dilation, Special Relativity, Metric, Schwarzschild solution, Temporal Manifold, Golden Ratio

\section{Introduction}

In a previous paper (Austin, 2017) a method for calculating time dilation from Newtonian gravitational potential energy (NGPE) provided a first order equivalence to Schwarzschild's solution to Einstein's field equations (Schwarzschild, 1916). The previous derivation followed a path of Special Relativity's derivation of relativistic kinetic energy by Resnick ( Halliday, Resnick, 1985). The paper did not provide a merger of kinetic energy and NGPE for the purpose of transforming the time component between observers. This paper will merge the two methods into a single transformation. From this transformation a topological space with a manifold and metric will be constructed.

\section{Combining NGPE and Relativistic Kinetic Energy}

In the previous paper on Newtonian gravitational potential energy (NGPE), Special Relativity's $\gamma$ was cast as a function of kinetic energy. 
(1)

$$
\gamma(v)=1+\frac{T(v)}{E_{0}}
$$

Where $\mathrm{v}$ is relative velocity, $\mathrm{T}$ is relativistic kinetic energy and $E_{0}$ is rest energy of the moving particle. $\mathrm{T}$ is:

$$
T=E_{0}(\gamma-1)
$$

A second equation derived from the previous paper for time dilation (based on NGPE) is

$$
\varphi=1-\frac{V(r)}{E_{0}}
$$

Where $\varphi$ is a scalar time transformation (unit time magnitude) from an infinite distance from the mass creating the NGPE $V(r)$ to a radial distance $r$ from the mass, and rest energy $E_{0}$. The NGPE is:

$$
V(r)=-\frac{G M^{2}}{r}
$$

Where $\mathrm{G}$ is the Newtonian gravitational constant, $\mathrm{M}$ is the central mass creating the gravitational potential, $\mathrm{r}$ is the radial distance from the center of $\mathrm{M}$.

The desire is to combine the NGPE effect on time (equation 3), with Special Relativity's kinetic energy derived effect on time (equation 1). Consider a function $\alpha$ based on relativistic kinetic energy and NGPE that transforms time between observers. A potential candidate for combined energy transformation constructed from equations 3 and 1 :

$$
\alpha(T, V)=1+\frac{T(v)}{E_{0}}-\frac{V(r)}{E_{0}}
$$

By redefining $\varphi$ from equation (3) to be: (the one is removed so to only calculate the change)

$$
\varphi=-\frac{G M}{r c^{2}}
$$

And substituting equations (1) and (3) into equation (5) then simplifying,

$$
\alpha(T(v), V(r))=\gamma-\varphi
$$

Where $\mathrm{v}$ is velocity about a fixed massive body, $\mathrm{r}$ is the radial distance from the center of the massive body, $\mathrm{T}$ is the kinetic energy function 
(from equation 2), $\mathrm{V}$ is the potential energy function (from equation 4), $\varphi$ is from equation (6), and $\gamma$ is Special Relativity's scalar as shown in equation (1).

Next, equation (7) needs equation (1) and (3) to align their effect on time. Recall from the first paper, the two time transformations; first is kinetic energy from equation (1), time transformation:

$$
t^{\prime}=\left(1+\frac{T}{E_{0}}\right) t=\gamma t
$$

Where the primed time is in the moving frame in Special Relativity. The second time transformation is based on NGPE from equation (3):

$$
t^{\prime}=(1+\varphi) t
$$

Where the primed time is within a gravitational potential and the unprimed is at an infinite distance from the mass generating the potential. As $\gamma$ increases, its effect on time is opposite the effect $\varphi$ has when it increases. Consider the following table:

Energy

$\mathrm{T}$ increases

$\mathrm{V}$ increase
Scalar Effect

$\gamma$ increases

$\varphi$ decreases
Time Dilation Eq.

$$
t^{\prime}=\gamma t
$$$$
t^{\prime}=(\mathbf{1}+\varphi) t
$$
Time interval increases as $\mathrm{T}$ increases Time interval decreases as $\mathrm{V}$ increases

Table 1

$\mathrm{T}$ and $\mathrm{V}$ have the opposite effect on time. By subtracting $\varphi$ in equation (7), it is modified to

$$
\alpha(T(v), V(r))=\gamma+\phi
$$

Equation (8) is used to transform the time component from a location with no relative kinetic energy and no gravitational potential to a location with one or both (relative kinetic energy and gravitational potential energy).

$$
t^{\prime}=\alpha(T(v), V(r)) t
$$

Where $t$ is the time component at the location with no kinetic energy and no gravitational potential. At this stage, equation (8) continues to have an issue, it combines inertial frames and accelerated frames in the time transformation scalar $\alpha$. This was briefly discussed in the previous paper, but no proper way to merge the two was contemplated. Next we consider a 
solution to this issue by building sets of observers from equations (1) and (3).

\section{Time Dilation Sets}

Consider the set $\mathcal{V}$ of all possible values of $T$ from equation (1):

$$
\mathcal{V}=\{T \mid T(v)\}
$$

The velocity has a range of zero to $\mathrm{c}$ (the speed of light in a vacuum) and $\mathrm{T}$ has a range from zero to infinity. Next, consider set $\mathcal{R}$ of all possible values of $\varphi$ from equation (3):

$$
\mathcal{R}=\{\varphi \mid \varphi(r)\}
$$

The radial distance $r$ has a range from zero to infinity and $\varphi$ has a range from zero to infinity.

Sets $\mathcal{V}$ and $\mathcal{R}$ have scalar elements representing kinetic energy magnitude and gravitational potential energy magnitude respectively. The union of the sets form set $\mathcal{E}$.

$$
\mathcal{E}=\{\mathcal{V} \cup \mathcal{R}\}
$$

Next, form a topological space $\mathrm{T}$ over $\mathcal{E}$. T contains $\mathcal{E}$ and the empty set $\varnothing$. On $\mathrm{T}$ consider a manifold $\mathcal{M}$. About every point $p$ on $\mathcal{M}$ there is a space near $p$ that is homeomorphic to Euclidean space of dimension $\mathcal{R}^{3}$ (Wolfram, 2017). Each Euclidean space about point $\mathcal{p}$ on $\mathcal{M}$ maintains the following axioms:

1. There is an observer at every $\mathfrak{p}$ on $\mathcal{M}$, for the purpose of measuring time and space without affecting what is being measured.

2. The unit time $\hat{t}$ is measured by each observer at their point $p$ on $\mathcal{M}$.

3. The unit length for the Euclidean space about point $p$ is dependent on unit time as measured at point $p$ on $\mathcal{M}$. The unit length is $c \hat{t}$. Where $\mathrm{c}$ is the speed of light in a vacuum and $\hat{t}$ is the unit time.

Each point on $\mathcal{M}$ is uniquely defined by an element from $\mathcal{V}$ and an element from $\mathcal{R}$ in $\mathcal{E}$.

Constructed is a topological space containing a manifold $\mathcal{M}$. The manifold follows axioms by which the local Euclidean space is formed. When considering the set of all observers $\mathrm{O}$, there are four subsets of interest.

\section{Sets of Observers}

Consider the following sets of observers:

$$
\begin{aligned}
& \text { set } \mathcal{A}=\{O \mid \mathcal{V}=0 \wedge \mathcal{R}=0\} \\
& \text { set } \mathcal{B}=\{O \mid \mathcal{V}>0 \wedge \mathcal{R}=0\} \\
& \text { set } \mathcal{C}=\{O \mid \mathcal{V}=0 \wedge \mathcal{R}>0\}
\end{aligned}
$$




$$
\operatorname{set} \mathcal{D}=\{O \mid \mathcal{V}>0 \wedge \mathcal{R}>0\}
$$

Set $\mathrm{A}$ is a boundary for the scalar alpha from equation (8). Alpha has a range from 1 to infinity, and any observer in set A has an alpha value of 1 . Set A contains all observers who have no gravitational potential and no kinetic energy (the relative velocity is zero; in Special Relativity this is the rest frame).

Set B contains the equivalence of a moving frame in Special Relativity. It is made up of all observers who are in motion relative to set A and have no gravitational potential.

Set $\mathrm{C}$ contains observers with relative velocity zero to observers in set $\mathrm{A}$, and are within a gravitational potential.

Set D contains all observers with relative velocity greater than zero to observers in set A, and are within a gravitational potential.

Each of these sets form a subset of $\mathcal{E}$. Of interest are two subsets of $\mathcal{E}$ constructed from these sets. The privo set $\mathbb{P}$ and the exemplar set $\mathbb{E}$.

$$
\begin{gathered}
\text { set } \mathbb{E}=\{\mathcal{A}\} \\
\text { set } \mathbb{P}=\{\mathcal{B}, \mathcal{C}, \mathcal{D}\}
\end{gathered}
$$

The exemplar set observers have no kinetic energy and no gravitational potential. The exemplar set represents the points on the manifold where an observer's unit time interval is a minimal compared to all other observers. In other words, the observer's clock runs fastest in the exemplar set when compared to any clock in the privo set. Thus the exemplar set has a minimal boundary for the magnitude of the unit time interval on the manifold.

Equation (8) has the framework to transform from any privo set to the boundary condition on the manifold, which is the exemplar set. Next a metric for the manifold is derived.

\section{Formation of a Metric Based on $\alpha$}

The space considered is a $\mathcal{R}^{3}$ space. The line element is

$$
d s=\sqrt{g_{\mu v} X^{\mu} X^{v}}
$$

Where $g_{\mu v}$ is the metric over the manifold, and $X^{\mu}$ and $X^{v}$ are contra variant vectors. The metric is to maintain the invariance of the speed light between all observers. Consider equation (8a) where $t$ is time in set $\mathbb{E}$. At the boundary in $\mathbb{E}$ on the manifold, set the unit length to:

$$
\hat{\lambda}=c \hat{\imath}
$$


Where $\widehat{\lambda}$ is the unit length at the boundary, $\mathrm{c}$ is the speed of light in a vacuum and $\hat{\tau}$ is unit time at the boundary. The unit length in equation (13) is constructed from the axioms of the manifold, where the unit time determines the unit length. The value of $\alpha$ (from equation 8 ) in $\mathbb{E}$ is 1 . Any transformation of unit length from the privo set to the exemplar set on the manifold is:

$$
c t^{\prime}=\alpha c \tau \quad \therefore c \tau=\alpha^{-1} c t^{\prime}
$$

Where the primed time represents the privo observer's time. With equation $(12,13,14)$ one can derive

$$
d s^{2}=g_{\mu v}(c t)^{\mu}(c t)^{v}=c^{2} \tau^{2}
$$

Equation (15) is the square of the line element on the manifold. Consider the metric $g_{\mu v}$ a tensor which takes two vectors as an input $(\mu$ and $v)$. Its form is:

$$
g_{\mu v}=\left(\alpha_{\mu}(\gamma, \phi) \alpha_{v}(\gamma, \phi) I\right)
$$

Where I is the identity matrix and the alpha functions (from equation 8) return a scalar value. From equation (15), and (16) a possible solution for $g_{\mu v}$ in Cartesian components is,

$$
g_{\mu v}=\left(\begin{array}{ccc}
\alpha_{\mu}(\gamma, \phi) \alpha_{v}(\gamma, \phi) & 0 & 0 \\
0 & \alpha_{\mu}(\gamma, \phi) \alpha_{v}(\gamma, \phi) & 0 \\
0 & 0 & \alpha_{\mu}(\gamma, \phi) \alpha_{v}(\gamma, \phi)
\end{array}\right)
$$

$$
=\left(\begin{array}{ccc}
\alpha_{\mu}(\gamma, \phi)^{-1} \alpha_{v}(\gamma, \phi)^{-1} & 0 & 0 \\
0 & \alpha_{\mu}(\gamma, \phi)^{-1} \alpha_{v}(\gamma, \phi)^{-1} & 0 \\
0 & 0 & \alpha_{\mu}(\gamma, \phi)^{-1} \alpha_{v}(\gamma, \phi)^{-1}
\end{array}\right)
$$

Of note, the diagonals always have the same value.

\section{Invariance of the Line Element}

The line element from equation (15) is derived from using a boundary condition length in equation (13). With the hypothesis all observers agree with the boundary length and the assumption nature behaves according to equation (15), an observer at a local point about the massive body will observe all measurements with a set of local basis vectors and unit time. The unit length at each location will be determined by the unit time from axiom 3: 


$$
\hat{l}=c \hat{t}
$$

Deriving the local unit length at a point on the manifold from the boundary length is

$$
\hat{l}_{1}=\alpha_{1}(\gamma, \phi) c \hat{\tau}
$$

Note at the boundary on the manifold $\hat{l}_{1}=c \hat{\tau}$.

The unit length at the boundary on the manifold as derived from a local unit length is

$$
\hat{\lambda}=c \hat{\imath}=\frac{\hat{l}}{\alpha(\gamma, \phi)}
$$

Where $\hat{\lambda}$ is the unit length at the boundary, $\hat{l}$ is the unit length at a point on the manifold. Thus any observer on the manifold agrees equation (21)'s $\hat{\lambda}$ value is invariant.

\section{Geodesic from the Lagrangian}

Equation (21) contains the basis component of a vector which transforms as a covariant as in equation (18) and a vector component would transform as a contra variant as in equation (17). Thus:

$$
d s^{2}=g_{\mu v} X^{\mu} X^{v}=g^{\mu v} X_{\mu} X_{v}
$$

The square of the line element for a basis vector is

$$
d s^{2}=g_{\mu v} X^{a} X^{b}=\alpha_{a}(\gamma, \phi) \alpha_{b}(\gamma, \phi)(c \hat{\tau})^{2}
$$

Converting equation (15) to spherical components

$$
d s^{2}=\alpha^{2} d r^{2}+\alpha^{2} r^{2} d \phi^{2}+\alpha^{2} r^{2} \sin ^{2}(\phi) d \theta^{2}
$$

With $r$ constant, the Lagrangian is

$$
L=\alpha^{2} r^{2} \dot{\phi}^{2}+\alpha^{2} r^{2} \sin ^{2}(\phi) \dot{\theta}^{2}
$$

Thus the action is

$$
S=\alpha^{2} r^{2} \int_{a}^{b}\left(\dot{\phi}^{2}+\dot{\theta}^{2} \sin ^{2}(\phi)\right) d \tau
$$

When $\dot{\theta}$ is zero, there is a minimum, planar motion. The action then is

$$
S=\alpha^{2} r^{2} \int_{a}^{b} \dot{\phi}^{2} d \tau
$$

Equation (27) continued: 


$$
S=\alpha^{2} r^{2} \int_{a}^{b} \dot{\phi}^{2} d \tau=\alpha^{2} r^{2}\left(\phi_{b}-\phi_{a}\right)
$$

Equation (28) is the geodesic equation for a fixed radius about a massive object using the metric from equation (17). In an elliptical orbit, $r$ can be given a dependency on the angle $\phi$ for an elliptical orbit as follows: (Wheeler, Misner, Thorne, 1970, p. 1110)

$$
r(\phi)=\frac{a\left(1-e^{2}\right)}{1+e \cos (\phi)}
$$

Where $a$ is the semi-major axis, $e$ is the eccentricity of the orbit and $\phi$ the angle of the orbit. $\alpha$ contains $\mathrm{r}$ within its definition, substituting equation (29) into the RHS of equation (28) and integrating over a full orbit (an orbit maintained by Newtonian classical gravitational force). The full rotation per orbit is

$$
\begin{aligned}
\text { radians }=\int_{0}^{2 \pi}\left(\frac{a\left(1-e^{2}\right)}{1+e \cos (\phi)}\right)\left(\frac{1}{\sqrt{\frac{G M}{\left(\frac{a\left(1-e^{2}\right)}{1+e \cos (\phi)}\right) c^{2}}}}\right. \\
\left.+\frac{G M}{\left(\frac{a\left(1-e^{2}\right)}{1+e \cos (\phi)}\right) c^{2}}\right)^{2} d \phi
\end{aligned}
$$

Equation (30) shows an advance for any orbit in a gravitational potential. Consider Mercury's orbit about the sun. Using (NASA, 2017)

$$
\begin{gathered}
G=6.67384^{-11} \\
c=299792458 \mathrm{~m} / \mathrm{s} \\
\text { Mass Of Sun }(M)=1.98892^{30} \mathrm{Kg}
\end{gathered}
$$

Semi Major Axis Mercury $(a)=57909100000 \mathrm{~m}$

Eccentricity of Orbit $(e)=0.206$

The perihelion of Mercury advances $5.02^{-7}$ radians per revolution. Over the course of a century, the advance is 42.98 arc seconds. This is in agreement with both observational and Schwarzschild's solution to Einstein's field equations (Einstein 1905). 


\section{Summary}

It has been shown a temporal manifold can be derived from Newtonian gravitational potential and Special Relativity. A metric was constructed from the invariant speed of light by all observers. From the metric a geodesic was derived and used to calculate an orbit about a central massive object. The results align well with observation and Schwarzschild's solution to Einstein's field equations.

This method is not to be considered a replacement for General Relativity but a compliment. This method provides a teaching path from classical Newtonian gravitational force to a minimum first order accuracy of General Relativity. The method can be used much in the same manner as a simple transformation is used in teaching Special Relativity without introducing Minkowski space.

\section{Of Note}

An interesting correlation (without comment) exist between equations (1), (3) and the golden ratio. If one sets equation (1) equal to equation (3) in a gravitational maintained orbit, the golden ratio appears. Setting these equal is when the temporal effect of kinetic energy is equal to the temporal effect of gravitational potential energy. Consider

$$
\sqrt{\frac{1}{1-\frac{G x}{c^{2}}}}=1+\frac{G x}{c^{2}}
$$

Where $\mathrm{x}=\frac{M}{r}$, the LHS of (31) is the kinetic energy effect from equation (1) when in an orbit at radial distance $r$. The RHS is from equation (3) and is the gravitational potential energy of an object in orbit at radial distance $r$. When $\mathrm{x}$ is solved for:

$$
x=\frac{c^{2}}{G} \frac{(\sqrt{5}-1)}{2}
$$

The golden ratio inverse is $\frac{(\sqrt{5}-1)}{2}$ and is an exact solution to equation (31). 


\section{References:}

1. Austin, Rickey (2017) Gravitational Time Dilation Derived from Special Relativity and Newtonian Gravitational Potential. European Science Journal

2. Schwarzschild K. (1916) ber das gravitationsfeld eines massenpunktes nach der einsteinschen theorie. Sitzungs-berichte der Deutschen Akademie der Wissenschaften zu Berlin

3. Halliday, David., \& Resnick, Robert.(1985) Basic Concepts In Relativity And Early Quantum Theory. John Wiley and Sons, Inc., 2 edition.

4. Wolfram (2017) Weisstein, Eric W. "Topological Manifold." From MathWorld--A Wolfram Web Resource. http://mathworld.wolfram.com/TopologicalManifold.html

5. NASA (2017) Williams, David R. Planetary Fact Sheets, NASA Goddard Space Flight Center

6. Wheeler, Misner, Thorne. (1970) Gravitation. W H Freeman and Company

7. Einstein (1905) Einstein, Albert. On the electrodynamics of moving bodies. Annalen der Physik 